\title{
Los catálogos de venta de las librerías argentinas \\ Eclosión y especialización de un medio publicitario efímero, 1850-1920
}

\author{
( Juan David Murillo Sandoval \\ Instituto Caro y Cuervo, Bogotá, Colombia | juan.david.murillo.s@gmail.com / https://orcid.org/oooo-0001-8295-2483
}

\begin{abstract}
Resumen
Los catálogos de venta de libros son una fuente principal en la historia del comercio del libro. Su razón publicitaria permite acercarse a los ritmos del mercado del libro, y orienta sobre las modalidades y estrategias usadas por los libreros para alcanzar sus clientelas. La condición efímera de estos materiales ha impedido, sin embargo, documentar su impacto en la vida comercial urbana o incluso determinar la frecuencia de su uso por parte de los libreros. Sobre una base de datos que registra más de 500 catálogos publicados para el espacio cultural argentino entre 1850 y 1920, este artículo identifica y analiza los momentos de auge de estas producciones, releva sus orígenes y emisores, y señala algunas de sus transformaciones en el tiempo. El estudio enfatiza así la necesidad de reconocer al catálogo de librería como un material primario en la consolidación de una cultura impresa en la Argentina.
\end{abstract}

\section{The sale catalogues of the Argentine booksellers: emergence and specialization of an ephemeral advertising medium, 1850-1920}

\begin{abstract}
Book sale catalogues are the main source in the history of the book trade. Its advertising nature allows us to approach the rhythms of the book market, and guides on the strategies used by booksellers to reach their customers. The ephemeral nature of these materials has prevented, however, to know their impact on commercial life or even determine the frequency of their use by booksellers. Based on a database that registers more than 500 catalogues published for the Argentine cultural space between 1850 and 1920, this article analyzes the rise of these materials, reveals their origins and producers, and highlights some of their transformations in a long time. The study thus emphasizes the need to recognize bookshop's sales catalogues as a leading material in the consolidation of a printed culture in Argentina.
\end{abstract}




\section{Introducción ${ }^{1}$}

1. Este estudio tuvo su origen en el proyecto doctoral: "Conexiones libreras. Modernización y cultura impresa entre Argentina, Chile y Colombia, 1880-1920", adelantado en el Instituto de Historia de la

Pontificia Universidad Católica de Chile y financiado por CONICYT (Beca Doctorado Nacional 201363130178). Enriquecida con nueva documentación, esta versión se enmarca en el proyecto "Historia del comercio de librería en América Latina, siglos XVIII-XX", respaldado por el Instituto Caro y Cuervo.

2. Pensamos aquí en proyectos pioneros de inventario analítico, como Esprit des Livres: Catalogues de vente de bibliothèques de l'époque moderne, liderado por Annie Charon en la École nationale des chartres (http://elec.enc. sorbonne.fr/cataloguevente/intro. php), y en el más reciente proyecto Mediate, coordinado por Alicia

C. Montoya desde la Radboud Universiteit (http://mediate18.nl/).

3. Este resulta particularmente visible en la creación de colecciones especiales, como el fondo Q10 de la Bibliothèque nationale de France $(\mathrm{BnF})$, pero ante todo en el inicio de nuevos proyectos que profundizan su estudio para entender la economía del libro en los siglos XVI y XVII (Granata y Nuovo, 2018), o la modernización de las estrategias de libreros y editores en el mercado hispanoamericano (Rueda Ramírez y Agustí, 2016; Agustí, Baró y Rueda Ramírez, 2018).

4. Una excepción la supone el Archivo documental de Gisbert y Cía. S. A. localizado en La Paz, Bolivia, que conserva la documentación perteneciente a la Librería Universitaria de Arnó Hnos. negocio creado a inicios del siglo XX y que fue sucedido por la familia Gisbert en la década de 1930 (Torrico, 2020).
A pesar de su incontestable importancia para la historia del libro, los catálogos de venta producidos por libreros y librerías se han convertido en objetos concretos de investigación solo en tiempos recientes. En general, la aparición y ejecución de proyectos dedicados especialmente a su identificación y análisis, así como al examen de su función dentro del mercado del libro y en la circulación de las ideas, cuenta algo más de una década, y todo a pesar de su repetido uso como fuente.

El interés por los inventarios y catálogos impresos de libros tiene, sin embargo, una historia más prolongada. Los estudios bibliográficos reportan una preocupación por estos materiales que recorre alrededor de un siglo, mientras que las últimas tres décadas han visto aparecer iniciativas orientadas exclusivamente a su recuperación, inventario y análisis. Repartidas entre distintas universidades, estas han tendido a privilegiar, empero, catálogos de venta distintos a los manejados por las librerías, como son los asociados con colecciones y bibliotecas privadas, cuya naturaleza engrana con otras instancias de comercialización libreril como las subastas y los remates.

Más allá de sus objetivos primarios, estos proyectos son hoy referencias obligadas en el estudio de los catálogos de librería, una vez han complejizado su entendimiento como materiales comerciales y bibliográficos claves para entender la formación de bibliotecas privadas y públicas de los siglos XVI y XVII, reconstruir el sistema literario europeo del siglo XVIII, o estudiar la misma actividad de sus productores, sean estos eruditos, bibliotecarios o mercaderes de libros ${ }^{2}$. En otras palabras, estas iniciativas han hecho evidentes las posibilidades del catálogo de venta de libros, sea o no producido por los libreros, para ampliar el conocimiento sobre la cambiante geografía del libro, sus agentes y públicos.

Indispensable para la historia económica del libro, el catálogo de librería se ha convertido, asimismo, en un material relevante para la historia intelectual y literaria, a la vez que su estudio puede favorecer la reconstrucción de redes académicas y eruditas, ayudar a rastrear procesos transferencia cultural, o inclusive servir a la caracterización de los agentes e instituciones mediadoras en la visibilidad y popularidad de géneros, títulos y autores determinados.

En esta orilla del Atlántico, los influjos de la historia del libro y la lectura tuvieron un impacto temprano en el interés por los catálogos de librería. Los estudios de Alejandro Parada (2002, 2007a, 2007b), en parte paralelos a los primeros proyectos europeos, demostraron la importancia del catálogo para analizar el paisaje de lecturas que rodeó a las élites del espacio rioplatense y permeó sus ideas y proyectos durante los primeros tiempos republicanos. No obstante, este importante capítulo de la historia del libro continental, bien abierto por Parada, no ha tenido la continuidad merecida. Mientras en Europa la investigación asentada sobre los catálogos de libreros y editores crece y se complejiza, el interés por estos mismos materiales en América Latina parece pausado ${ }^{3}$. La casi total ausencia de archivos pertenecientes a los libreros, junto con la irregular conservación de los catálogos de venta de las librerías en las bibliotecas nacionales y públicas que están en la base de este problema, pero no excusan la desatención dada a estos materiales y, en definitiva, a la historia del comercio de librería en el continente ${ }^{4}$. Una historia que, como los mismos catálogos evidencian, fue característicamente conectada y transnacional.

Con miras a reavivar la atención sobre los catálogos de librería producidos y circulados en el continente, este artículo realiza un estudio cuantitativo de la presencia de estos medios publicitarios en el espacio cultural argentino entre 1850 y 1920. En específico, se busca destacar los momentos de auge de estos materiales, identificar sus lugares de 
producción y principales emisores, y señalar algunas de sus mutaciones más notorias para un periodo extenso y complejo, atravesado por los procesos de construcción estatal y por transformaciones demográficas, socioculturales y económicas sustanciales. Se trata, además, de un periodo que vio la afirmación y expansión de la actividad librera en la Argentina y, con ella, la eclosión del catálogo como medio publicitario.

Gracias a las disecciones realizadas por Parada a los catálogos de Duportail Hermanos (2002) y Marcos Sastre (2007b), sabemos que estos materiales hicieron parte del paisaje mediático porteño desde inicios de la vida independiente. Durante la época de Rosas, sin embargo, su producción parece detenerse. Si bien no fue un momento de parálisis en la actividad librera, ningún catálogo ha podido hallarse para la década de 1840 , y apenas 4 se han identificado para la siguiente, todas piezas que denotan una esperable dependencia de las librerías porteñas con respecto del mercado internacionals.

Entrada la década de 1860, la presencia de los catálogos se triplica con 12 folletos, número que, pese a mantenerse bajo, documenta el uso transversal del recurso por parte de librerías como la "Española" de Teodomiro Real y Prado, la "Americana" de Eduardo Halbach, la Librería de Juan C. Ure, la Imprenta y Librería de Mayo de Carlos Casavalle, y la Librería de Eudoro Carrasco en Rosario, casa que produjo el primer catálogo observado fuera de Buenos Aires ${ }^{6}$. Con todo, el uso del catálogo se dispara, según veremos, en las dos décadas siguientes, ilustrando un auge que responde al aumento de las librerías en Buenos Aires, un total de 70 para 1886 según la Gran guía de la ciudad de Hugo Kunz (1886: 912), y que conecta con la formación del mercado editorial local (Pastormelo, 2005; Sorá, 2011)7.

La prolongación de este análisis hasta 1920 responde, en esta medida, a la necesidad de explorar la vida de los catálogos de venta de libros en un momento en el que las condiciones de posibilidad para la producción, el comercio y la publicidad del libro en la Argentina mutan nuevamente. Como se verá, la presencia de los catálogos se reduce luego de 1910 -hecho que indica, por cierto, una parálisis en su conservación-, pero aquellos identificados destacan también por provenir sobre todo de editores o librero-editores que de libreros en singular.

Se trata, ciertamente, de una época de relevo entre los dinamizadores del mercado, pero también de un nuevo contexto mediático que exigía también nuevas estrategias de mercadeo del libro. Conducir este estudio entre el momento de reactivación del catálogo hacia 1850 y un nuevo punto de quiebre durante el decenio de 1910 permite, pues, no solo tener una mirada de amplia duración a la vida local de un producto singular por su razón comercial y naturaleza momentánea, sino abrir hipótesis sobre la evolución del quehacer librero al calor de mutaciones socioculturales más generales.

Con relación a la muestra usada en este estudio, esta se sustenta en la base de datos Catálogos Libreros Latinoamericanos (CLL), resultado indirecto del proyecto Conexiones libreras: modernización y cultura impresa entre Argentina, Chile y Colombia, 1880-1920 (2013-2019), y que cuenta con cientos de registros de catálogos publicados o manejados por librerías de todo el continente. Dado su imprevisto desarrollo, una breve semblanza de este corpus se hace necesaria. Formado primero con datos extraídos de los catálogos de la Biblioteca Nacional Mariano Moreno (BNMM), la Biblioteca Nacional de Maestros (BNM), el CeDInCI, la Biblioteca Nacional de Chile (BNCh) y la Biblioteca Nacional de Colombia (BNC), su objetivo consistía en registrar y comparar el volumen de catálogos de venta publicados en Argentina, Chile y Colombia entre 1880 y 1920, así como en identificar los establecimientos más interesados en su uso.
5. Se trata del Catálogo de libros selectos y estampas que se hallan de venta en Buenos Ayres de la casa parisina de Rosa y Bouret (1850), el Catálogo general de los libros que se hallan en la Librería Nueva de Carlos Alou (1854), el Catálogo de las obras de suscripción y venta en la Librería Ortiz (1855) y el doble Catálogo de libros españoles y franceses de la Librería de la Victoria (1856). Estas cuatro piezas se registran o encuentran, respectivamente, en la revista La Literatura Argentina (1932, No. 52: 392), el catálogo de la Biblioteca del Museo Mitre (Topográficos: 46, 1, 22b y 44, 1,5 d) y en la Sala Medina de la Biblioteca Nacional de Chile.

6. Resguardado en la Biblioteca Nacional Mariano Moreno, el Catálogo de libros en castellano, francés, latín, e inglés que se hallan a venta en la Librería e Imprenta de Eudoro Carrasco (1864) es un ejemplo temprano de la expansión de la actividad librera en el interior del país. En sus cincuenta páginas, este expone vínculos comerciales con casas parisinas, inglesas, y barcelonesas, ofreciéndose además como una confiable casa de surtido de maquinaria y útiles de imprenta y escritorio.

7. En 1855 se contaban 11 librerías en Buenos Aires (Almanaque comercial y guía de forasteros, 1855: 116) y en 1869 un total de 28 (De la Fuente, 1872: 70), por lo que la cifra reportada por Kunz en 1886 manifiesta una multiplicación por 2,5 en el número de negocios en poco más de 15 años. 
8. Algunas bibliografías internacionales fueron también útiles, como la Guide to Law and Legal Literature of Argentina, Brazil and Chile (1917) de Edwin M. Borchard, e incluso algunas más contemporáneas como la Bibliografía de la ciudad de Buenos Aires (200o) de Eduardo Luis Criscuolo

9. El desprecio hacia cierto tipo de catálogos fue público por parte de bibliógrafos como Alberto Navarro Viola, quien en su Anuario Bibliográfico de 1881 se refirió a un catálogo de la librería de Ángel Estrada como una pieza de "poca importancia", formada por "obras de escuela y de prácticas religiosas en su mayor parte" (1881: 274). Los límites de las bibliografías los anticipa también su propia

inconstancia, pues entre el Anuario de Navarro Viola y el Boletín de la Biblioteca Pública en La Plata se eleva un vacío de más de un decenio sin publicaciones de corte bibliográfico en la Argentina.
Para adelantar comparaciones rigurosas, sin embargo, este primer boceto de base de datos mostraba inconvenientes, ya que los catálogos existentes en las bibliotecas no destacaban por su abundancia, de manera que su conteo podía dar una idea equivocada sobre la producción de estos efímeros y su real presencia en la vida comercial urbana. Frente a esta dificultad, se optó por explorar nuevos repositorios y acudir a bibliografías que pudieron recoger catálogos que no lograron perdurar hasta hoy. El Anuario bibliográfico de la República Argentina (1879-1887) de Alberto Navarro Viola fue así clave para identificar piezas emitidas por las principales librerías bonaerenses de la década de 1880. El Boletín de la Biblioteca Pública de la Provincia de Buenos Aires en la ciudad de La Plata (1899-1905) hizo lo propio para inicios del siglo XX, mientras que varias entradas de La Literatura Argentina (1932) sirvieron para ampliar el corpus o corroborar datos ${ }^{8}$.

La información extraída de estas publicaciones permitió ampliar el volumen de catálogos identificados y percibir mejor la naturaleza constante de muchos de ellos. Cotejada y complementada con materiales fichados en bibliotecas estadounidenses y europeas, así como con registros provenientes de instituciones como el Museo Mitre, la base de datos logró consolidar un corpus amplio y propicio para un primer análisis cuantitativo. Hasta el momento, este reúne 653 registros de catálogos para el caso argentino entre 1824 a 1954, pero como ya se ha indicado, para este estudio la muestra se limitó al periodo comprendido entre 1850 y 1920, rango que agrupa 544.

El avance en la identificación no ha implicado, con todo, la superación de las lagunas. Al depender de los registros provenientes de bibliografías, los cuales suelen entregar datos apenas titulares, mucha información útil para el estudio de los catálogos, como el número de libros anunciados, las formas de organización o la evolución de los precios, quedan necesariamente al margen. Es claro, a su vez, que las bibliografías no dieron cuenta de todo el universo de catálogos producidos. Si las bibliotecas y sus bibliotecarios se mostraron por lo general desinteresados en su control e inventario, los bibliógrafos tendieron a valorar únicamente los catálogos provenientes de las librerías más sobresalientes o próximas a sus propias trayectorias intelectuales. En otras palabras, la sociabilidad de librería derivaba en preferencias y prácticas que, al privilegiar a ciertos libreros y catálogos, relegó de las bibliografías aquellos folletos manejados por librerías pequeñas, obreras o populares?.

Conformada pues por catálogos existentes y consultables, pero ante todo por registros de materiales que no alcanzaron a conservarse en ningún lugar, la base de datos que sustenta este trabajo debe considerarse todavía aproximativa frente a las publicaciones ínsitas al oficio librero. No obstante, como lo recalca Roger Chartier, los historiadores "no se resignan fácilmente a no saber nada, a no decir nada o a no imaginar nada de las obras de las que solo conocen el título y, a veces, el nombre del autor" (2012: 16). Así, pues, creemos que los datos reunidos permiten acercarse a la vida de estos impresos efímeros y, mediante ellos, a la historia de las librerías en la Argentina.

Con base en lo anterior, el artículo abre con un breve recorrido por la historia del catálogo de librería, destacando lo que sus elementos más básicos aportan para el estudio de los libreros. Abocado ya al análisis cuantitativo, el apartado siguiente examina la producción de catálogos en la Argentina para el periodo descrito, identifica sus momentos de apogeo y declive, y la compara con otros casos nacionales. El tercer apartado se concentra sobre los libreros más activos en el uso del catálogo y establece una relación entre el vigor de su actividad publicitaria y sus conexiones internacionales, y el cuarto rastrea algunas de las mutaciones más notables en las formas asumidas por estos materiales. A manera de cierre, se recalca la necesidad de recuperar y poner en valor los catálogos de venta para avanzar en una historia no solo mejor documentada sino también conectada del comercio de librería continental. 


\section{Los catálogos de librería: precisiones y consideraciones}

Atada al inicio y desarrollo del comercio de libros impresos en Europa, la historia del catálogo de librería comienza a finales del siglo XV. Deudor de antiguas formas de publicidad asociadas con el libro manuscrito, pero dueño de las nuevas posibilidades que brindaba el cambio tecnológico y la lenta normalización del nuevo mercado de impresos, el catálogo de venta constituyó un segundo producto publicitario en la experimentación llevada a cabo por los libreros para posicionar el nuevo producto. Según Coppens y Nuovo (2018), los libreros produjeron primero hojas publicitarias con las que buscaron destacar las cualidades del libro impreso por sobre el manuscrito, sobre todo en lo concerniente a su legibilidad y condición "corregida" frente a los posibles errores de los copistas.

El primer boceto de catálogo surgiría, de hecho, en la forma de estas hojas, aunque abandonaría las fórmulas de persuasión. Ideado por Aldo Manucio para su establecimiento veneciano, este primer catálogo de librería, publicado en 1498 y reimpreso en varias ocasiones, marcó su primera gran distinción al concentrarse en listar los títulos disponibles en el taller y fijar sus precios (Coppens y Nuovo, 2018: 148). No obstante, el primer catálogo de venta en la forma que hoy lo conocemos, es decir, como un folleto (booklet) de relativa extensión, apareció décadas después en un contexto comercial que atestiguaba la expansión del mercado del libro y su conversión en un negocio de venta al por mayor. Fechado en 1537, su fabricación se debe a los herederos del impresor Johann Froben, de Basilea, y marca un debut en el uso de este formato en el centro y sur de Europa durante el siglo XVI.

Paulatina, de todos modos, la producción de catálogos no solo atestiguó el crecimiento de la producción impresa en la temprana época moderna, sino también la nueva geografía del mercado, la multiplicación de los agentes involucrados y sus clientelas, e incluso la consolidación de centros de comercio e intercambio especializados, como la Feria de Frankfurt (Nuovo, 2013). Al ritmo de estas mutaciones, el catálogo de librería se volvería también el recurso ideal para formar bibliotecas y colecciones privadas.

Con los siglos, el uso del catálogo por parte de libreros y librerías se hizo regular tanto en Europa como en América y otras regiones del globo. Para el caso suramericano, una vez finalizadas las revoluciones de independencia e iniciados los proyectos republicanos en las primeras décadas del siglo XIX, era previsible que este emergiera como medio de publicidad una vez que las primeras librerías aparecieran. No obstante, antes de la puesta en marcha de negocios exclusivamente avocados al comercio de libros, este era ya ejercido por toda una diversidad de comerciantes para quienes la publicación de catálogos de venta no era una prioridad.

En lo fundamental, estos negocios misceláneos, correspondientes a tiendas, casas introductoras y a veces a talleres de imprenta, limitaban su publicidad a la emisión de prospectos sueltos y la inserción de anuncios en las gacetas locales. Estos últimos, que bien podían consistir en un listado de títulos con precios, no cobijan el interés de este trabajo y, por tanto, no forman parte de la base de datos CLL. A pesar de su relevancia, los anuncios de prensa no son propiamente materiales de circulación autónoma ni brindan información suficiente sobre los libreros. Los catálogos de venta publicados en la forma de folletos sí patentan estas singularidades y sugieren, por lo demás, la presencia de negocios tendientes a privilegiar al libro como bien de consumo.

Además de ser asaz informativos sobre el surtido de los libreros, estos catálogos favorecen el rastreo de distintos aspectos propios al desenvolvimiento de las librerías, la mayoría de los cuales trasciende el alcance de cualquier anuncio. Tres aspectos peritextuales, asociados con la presentación de los catálogos, sirven para dar cuenta 
Imagen 1. Condiciones de venta en el Catálogo de la Librería Europea. Libros americanos modernos. Buenos Aires: Imprenta de Pablo E. Coni, 1882.

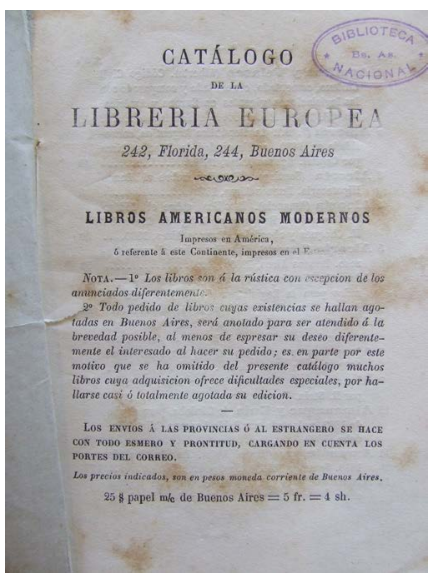

Fuente: BNMM, Colecciones especiales
10. Gracias al trabajo de Lima González Bonorino (2005), sabemos que sobre esta tradicional calle se asentaron también, para la década de 1860, las librerías "Nacional" de Martín Cosme, "Madrileña” de Claudio Lesarcegui, y "Buenos Aires" de Manuel Carrillo, tiendas de las que no se pudieron hallar catálogos.

11. Véanse: Catálogo de libros de la Librería de Ángel Estrada. Especialidad en toda clase de efectos escolares. Casa introductora de artículos de librería, escritorio y educación, Buenos Aires, Imprenta de Martín Biedma, 1883; y Catálogo de la Librería Europea de Jacobsen E Söderstedt, Buenos Aires, s.n., 1874

12. Almanaque para 1871. Catálogo de la Gran Librería y Papelería de C. M. Joly, sucesor de Lucien, París, Imprenta general de Ch. Lahure, calle de Fleurus, 9, 1870, 16-28. de esto. En primer lugar, y aunque parezca obvio, los catálogos ayudan a identificar a los libreros y situarlos en un mapa. La revisión sistemática de un elemento visible desde las portadas permite, sin embargo, ir allende la ubicación para observar la movilidad de los libreros por la malla urbana o incluso establecer quiénes les suceden y desde cuándo. Es decir, apenas con sus datos liminares, estos materiales permiten rastrear los ciclos de las librerías, sus propietarios y transformaciones.

Así, por ejemplo, la ajetreada vida librera de las porteñas calles de Victoria y Florida puede seguirse a través de los catálogos. La primera mantuvo por más de tres décadas a la librería de Claude M. Joly, sucesor de H. Lucien. Ubicada primero en el número 119, se desplazó después al 135-143 y, hacia el final del siglo XIX, al 719-721, dirección mantenida por los nuevos sucesores Prudent y Moetzel ${ }^{10}$. La Librería Francesa de Espiasse y Escary hizo lo propio sobre los números 73-75 hasta la separación de sus socios en 1883 . Joseph Escary continuaría sobre la estratégica arteria, situado sobre el 619, pero Espiasse pasaría a fundar su nueva Librería Céntrale sobre el número 10 de Florida, calle donde también relucieron, entre otros, las tiendas de Louis Jacobsen, ubicado en el 178 y después en el 242-244; Alfredo Brédalh, en el 234; Gustavo Mendesky, en el 359; Alfredo Cantiello, en el 26, y Juan Roldán, en el 436.

En segundo lugar, y una vez volteadas las portadas, las páginas de "Condiciones" o "Advertencias" surgen como un aspecto que avanza sobre las modalidades de venta. Los perfiles de las clientelas o la posición de los libreros en el mercado también se sugieren en estos apartados. Así, mientras catálogos como los del librero católico Ángel Estrada mostraban una expresa orientación hacia el ámbito educativo insistiendo que el negocio contaba con "toda clase de efectos escolares", los de Jacobsen exponían que este estaba en capacidad de alimentar toda clase de "bibliotecas particulares y públicas", e incluso a los "libreros por menor" ${ }^{11}$. (Imagen 1). Esta efigie de librero comisionista y gran distribuidor fue también replicada por Joly en sus catálogos-almanaques, medio donde prometía rebajas "A los provincianos" $y$ "Las personas de la campaña" que quisieran hacerse de una buena biblioteca ${ }^{12}$.

No obstante, algunos libreros-editores especializados, como Félix Lajouane, prescindían de las advertencias. En particular, este librero prefería explicitar las condiciones de venta solo de aquellas obras o revistas que editaba o importaba en exclusividad. Así lo hizo en 1897 con La Biblioteca de Paul Groussac, cuyas condiciones abrieron su catálogo de abril. Acompañadas con un prospecto de la revista, juicios de la prensa y un índice de sus primeros nueve números, las condiciones exponían el precio de la suscripción por un año, con las respectivas diferencias para Buenos Aires, las 


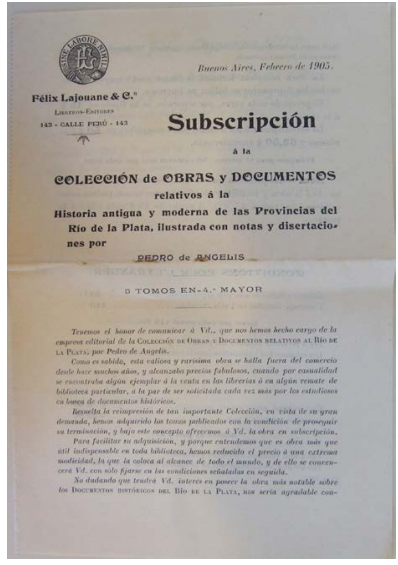

Imagen 2. Volante de suscripción inserto en el Catálogo de las Obras Editadas por Félix Lajouane \& Ca Libreros-editores, No. 76, enero de 1905. Buenos Aires: Lajouane, 1905.

Nota: Como se observa, el volante trata del proyecto de reedición de la Colección de obras y documentos relativos a la historia antigua y moderna de las provincias del Río de la Plata de Pedro de Angelis. La hoja señalaba el carácter que tendría la edición y detallaba las condiciones de suscripción en español y francés. provincias y el extranjero, así como el valor de un número suelto ${ }^{13}$. Cabe decir que Lajouane anexaba a sus catálogos volantes de suscripción a distintas obras que, al poder circular luego de forma independiente, extendían el alcance de su propaganda (Imagen 2).

Un tercer aspecto lo conforman los índices. Puestos al comienzo o el final de los folletos, unas veces generales y otras detallados, estos permiten seguir la evolución de la oferta o indicar giros en las demandas lectoras, a la vez que patentan el aumento de las divisiones temáticas o la aparición de secciones novedosas. Catálogos de librerías poco estudiadas, como la de Juan Bonmatí sirven para ilustrar esta dinámica. En uno de ellos, fechado en 1901, se evidencian secciones poco habituales, como la de "Sport, caza, equitación, gimnasia, juegos y tauromaquia", y una cierta reconversión de divisiones tradicionales, como la de artes y oficios, que tuvo por encabezado la acepción "Tecnología". De portada orlada, este catálogo anunciaba 4886 títulos disponibles y contó además con un índice alfabético de los autores incluidos (Imagen 3). Dirigido a lectores de cierta capacidad adquisitiva, pues era vendido a \$3 pesos, esta pieza documenta el perfeccionamiento de las prácticas catalogadoras entre los libreros, al tiempo que ilustra innovaciones en los géneros comerciados y sus fórmulas de publicitación ${ }^{14}$.

Por fuera de los tres aspectos descritos, y someramente ejemplificados, las listas de autores y títulos representan, claro está, la carne de los catálogos. No hace falta insistir sobre todo lo que su revisión puede aportar al estudio de la circulación de un título o autor, o de la constancia o irrupción de ideas políticas y estéticas. Pero en lo que concierne a este trabajo, es la cuestión de la periodicidad la que se impone como un último aspecto de relevancia, y tanto por lo que implica para la correcta identificación de los catálogos como para la reconstrucción del trabajo propagandístico de los libreros.

A pesar de que las bibliografías no lo indican, y de que los catálogos sobrevivientes en las bibliotecas no siempre lo hacen evidente, cualquier revisión a la vida de estos efímeros encuentra que su emisión pudo alcanzar ritmos muy constantes, entre quincenales, mensuales o bimensuales. En muchos casos, la inclusión de una numeración o la mención de la condición mensual en las portadas han hecho posible reconstruir la presencia de decenas de materiales a través del cotejo entre registros. No siempre este ejercicio pudo realizarse, según se verá, pero el mismo ha servido para documentar la larga trayectoria de numerosos catálogos periódicos, muchos no siempre entendidos como tales, como los almanaques, boletines y revistas
13. Ver: Catálogo de las obras publicadas por Félix Lajouane, Librero-Editor. No. 65, abril de 1897, Buenos Aires, 1897.

14. Aunque la agrupación dio cuenta de muchos tratados y manuales de oficios tradicionales, la noción de "Tecnología” parecía referir especialmente a una serie de tratados y enciclopedias dedicadas a técnicas modernas de construcción y a la fotografía. Ver: Catálogo de la Librería de J. Bonmatí, Buenos Aires, s.n., 1901. 
Imagen 3. Portada del Catálogo de la Librería de J. Bonmatí, Buenos Aires, s.n., 1901

Fuente: BNMM, Colección general

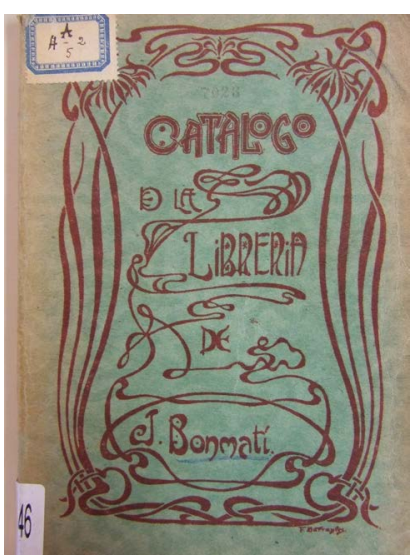

bibliográficas, todos productos menudos y generalmente gratuitos que, a la par que anunciaban libros y revistas, incluían informaciones útiles o breves reseñas literarias. Como veremos en el último apartado, la frecuencia de esta tipología de catálogos puede contarse entre los elementos que naturalizaron el comercio de librería entre los lectores argentinos.

\section{Ritmos, picos y espacios de producción}

Dado el tamaño de la muestra, algunos indicadores generales resultan útiles para introducir la dinámica de los catálogos de librería dentro del espacio argentino. De acuerdo con los datos recopilados, los años de mayor presencia de catálogos son 1879 y 1901 con 38 y 34 registros respectivamente. Les siguen $1878,1883,1900$, 1902 y 1903 con más de 25 registros. Otros años de mediana densidad reportaron entre 10 y 20, y los de menos entre 1 y 9. Para los años 1890, 1908 y 1909 no se han identificado catálogos. Sumados estos vacíos, el promedio anual de catálogos registrados entre 1850 y 1920 es igual a 9, y entre 1870 y 1910, periodo de mayor frecuencia, a 12.

Si graficamos estas cifras es posible observar otras dinámicas. La Gráfica 1 precisa la evolución por décadas y permite abrir el análisis de los auges y declives en la presencia de los catálogos. Lo que resalta a primera vista es, en efecto, el alza de la década de 1870 , pues se describe una notable multiplicación del número de catálogos registrados. Esta se reduce levemente en la década de 1880, cae más de la mitad para la siguiente y repunta en el primer decenio del siglo XX. Como se señaló antes, la caída de la época del centenario puede deberse a múltiples razones. Por un lado, ya no es el librero sino el editor quien empieza a comandar el paisaje del libro, y este prefiere anunciar en la prensa y las revistas de alto tiraje o difundir su catálogo en las contratapas de los libros que conforman sus colecciones. Por el otro, deben considerarse los efectos de la Gran Guerra y su impacto entre las librerías más dependientes del mercado internacional. 
Gráfica 1. Registro de catálogos de librería: evolución por décadas, 1850-1920

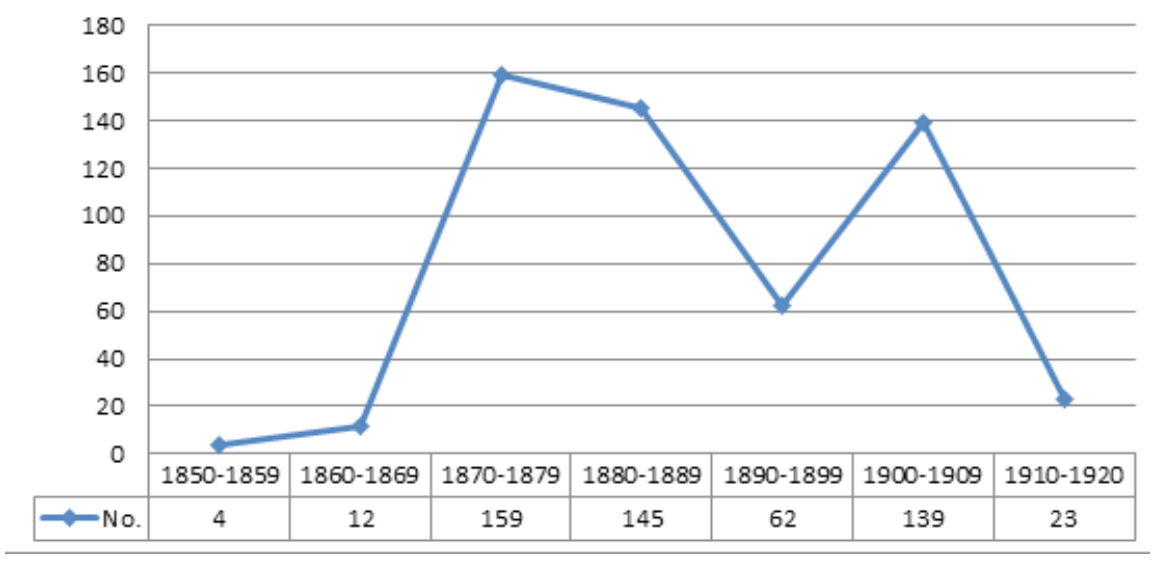

Fuente. Elaboración propia a partir de la base de datos CLL

Al discriminar las cifras por año surgen nuevos elementos. El alza en el primer lustro del siglo XX es clara, pero asimismo lo es la mayor constancia de los catálogos en las décadas de 1870 y 1880 , momentos en que el uso de este efímero publicitario conectó con las políticas de instrucción pública, la expansión de las actividades universitarias y los primeros impulsos dados a las bibliotecas populares (Gráfica 2) ${ }^{15}$. Al igual que en otros países, existe una relación entre la estructuración de un sistema educativo y la extensión del comercio librero, un fenómeno que en el caso argentino puede verificarse en las licitaciones del Consejo Nacional de Educación y seguirse en revistas como el Monitor de la educación común, pero asimismo palparse en la aparición de catálogos escolares, como el intitulado Textos de enseñanza primaria y secundaria que se encuentran en venta en la casa de Anjel [sic] Estrada (1883) o el Catálogo de los libros de enseñanza primaria y secundaria. Gran surtido de útiles necesarios para escuelas y colegios (1883) de la Librería del Colegio, y la normalización de secciones dedicadas a los "libros para premios" o colecciones de vulgarización científica.

Gráfica 2. Registro de catálogos de librería: evolución anual, 1850-1920

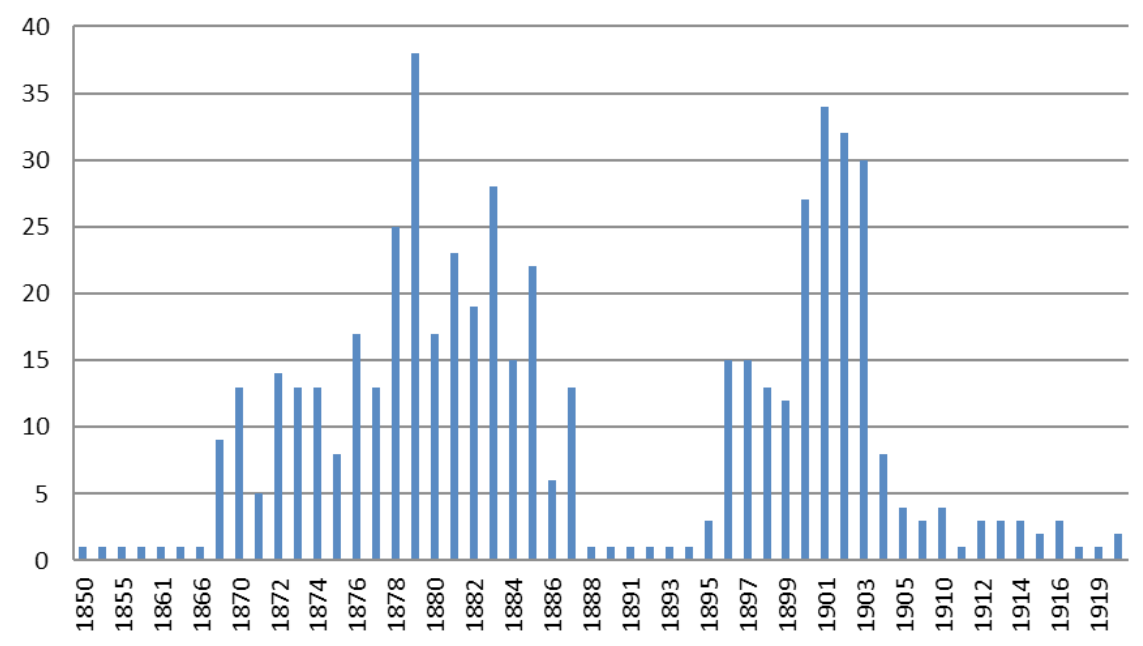

Fuente: Elaboración propia a partir de la base de datos CLL
15. Sobre los beneficios que reportaba el abastecimiento de las Bibliotecas Populares, véase el trabajo de Javier Planas (2017: 154-168) 
Volviendo a las cifras en su detalle anual, la baja de la década de 1890 puede explicarse en virtud de la crisis económica que afectó la Argentina en este periodo, y que se tradujo en numerosas quiebras, una alta desocupación, la baja del salario real, la reducción de los valores importados e incluso el freno de la migración (Sabato, 2012: 333). Siempre sensibles a las crisis, las librerías sintieron el golpe de la recesión y de los conflictos políticos paralelos, aunque hace falta una mayor inmersión investigativa sobre este periodo para establecer en qué medida sus operaciones se vieron afectadas.

Otra variable que puede examinarse gracias a la base de datos es el lugar de impresión de los catálogos. Buenos Aires es la ciudad donde se publican el mayor número (330), seguida de París (195). No obstante, los datos describen que el peso de la capital francesa se concentra en la década de 1870, momento donde los boletines de las librerías de Joly y Jacobsen son los más constantes (Gráfica 3).

Gráfica 3. Lugares de impresión de los catálogos 1850-1920

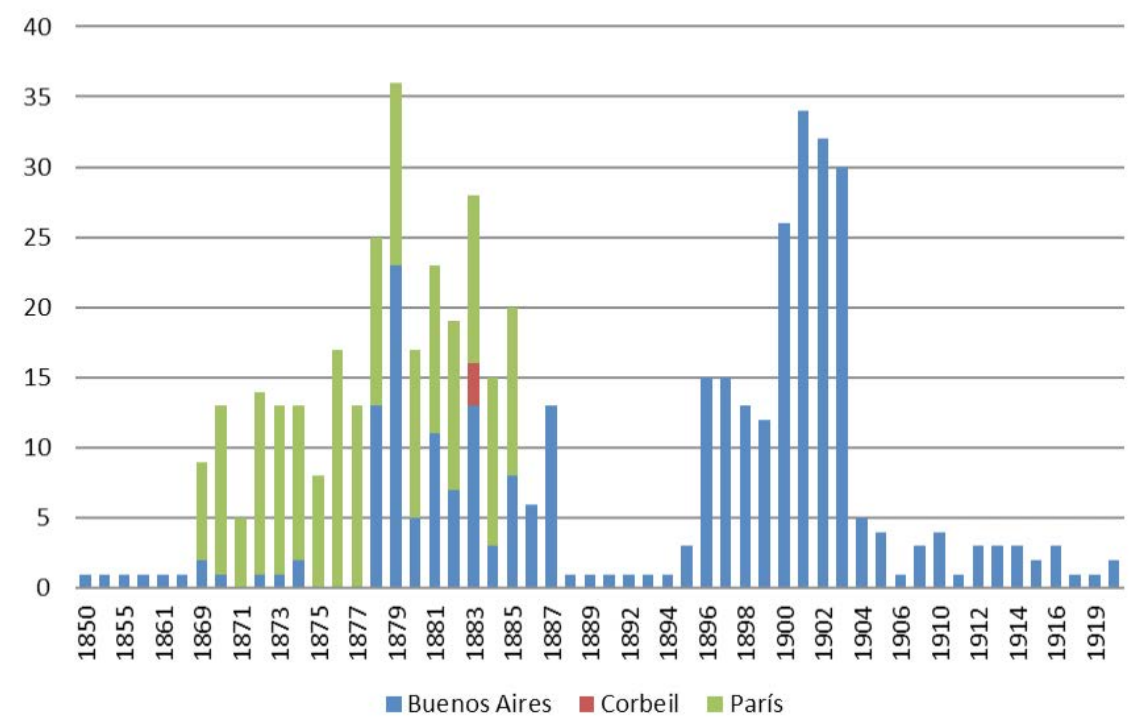

Fuente: Elaboración propia a partir de la base de datos CLL

Otras ciudades donde se publicaron catálogos fueron Rosario $(1,1864)$, Barcelona $(1,1885)$, y La Plata $(1,1900 ; 3,1904)$.

En concreto, es a partir de 1877 que los pies de imprenta comienzan a patentar un recambio. Gracias a la modernización de los talleres bonaerenses, los libreros pasaron a producir sus catálogos localmente, decisión que revela cambios en las formas en que las librerías interactuaban con el mercado europeo y en su propia valoración de la producción intelectual argentina. En efecto, ya no tenemos solo librerías dependientes de casas como Rosa y Bouret, sino negocios con vínculos transnacionales diversos y además comprometidos con los círculos letrados locales. El aumento de la producción nacional de catálogos señala, en definitiva, la cada vez mayor necesidad por parte de los libreros de controlar sus órganos de publicidad, fijando formas de presentación y organización orientadas a sus clientelas nacionales o a promover las obras de los hombres y mujeres de letras que empezaban a editar.

Para cerrar estos indicadores, una comparación entre los catálogos de las librerías argentinas con los de sus pares en Chile y Colombia resulta apropiada para dimensionar mejor la dinámica de estas publicaciones. Más allá de las diferencias cuantitativas, 
la Gráfica 4 permite inferir que la mayor o menor presencia de catálogos puede responder tanto de momentos de crecimiento o crisis económica como de coyunturas políticas locales. Chile muestra, por ejemplo, un crecimiento sostenido en la producción de estos materiales hasta 1891, año de la Guerra Civil, el cual se recupera con el fin del conflicto para volver a caer en coincidencia con la Gran Guerra.

Gráfica 4. Catálogos de librería en Argentina, Chile y Colombia: evolución por décadas, 1850-1920

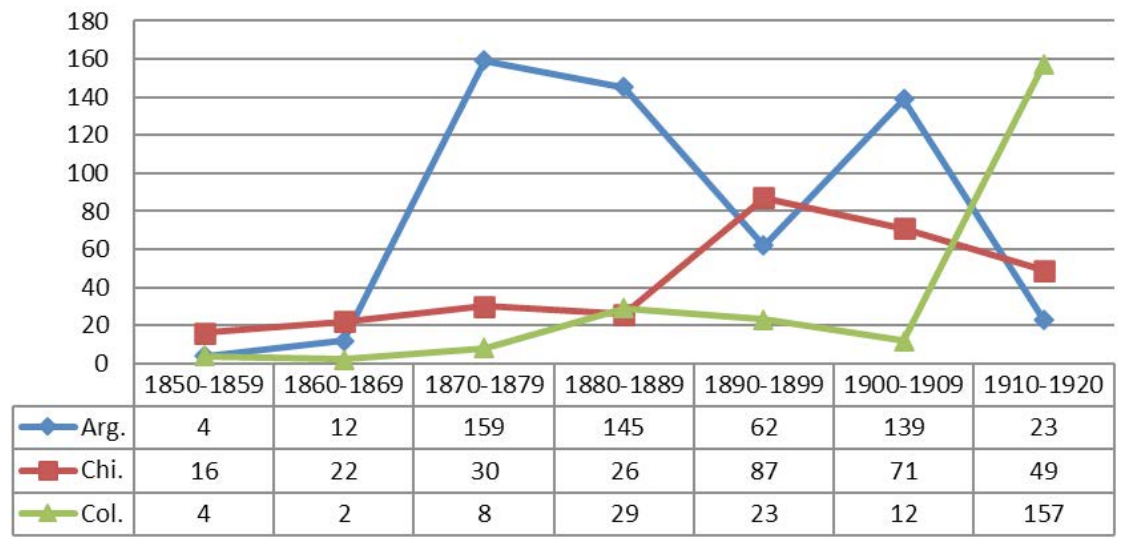

Fuente. Elaboración propia a partir de la base de datos CLL

En Colombia, la presencia del catálogo luce estancada en las primeras décadas del conteo, expandiéndose solo en la década 1880 para alcanzar un primer gran pico después de 1910, momento en el que incluso supera la cuantía vista en Argentina y Chile $^{16}$. No son pocas las variables que pueden explicar el lento despegue de la actividad librera en este país, pero la posición interior de su capital y las frecuentes guerras civiles de la segunda mitad del siglo XIX, que solían por supuesto interrumpir el movimiento cultural y comercial, son quizás las más importantes. Los picos coinciden, por lo demás, con momentos de paz relativa y alzas en el precio del café.

Dejando atrás estas consideraciones, la comparación sugiere, ante todo, que el fenómeno de eclosión de los catálogos no fue exclusivo de la Argentina. También en Chile y Colombia, como otros países del continente, los libreros y sus órganos aportaron a la expansión de una cultura de lo impreso y, con ello, a la formación de sociedades cada vez más mediatizadas. La comparación constata, por otro lado, y a pesar de los límites de los datos, que es posible observar tendencias y ensayar hipótesis. Como nos recuerdan Annie Charon y Elisabeth Parinet (2000), la rareza, delgadez y negligente fabricación en papel barato de los catálogos, que explica su difícil perduración, no reducen para nada su potencia documental.

\section{Libreros, publicidad y conectividad}

Dirijamos ahora la mirada a los libreros: ¿Quiénes fueron los principales responsables de la edición y puesta en circulación de catálogos en la Argentina? ¿Qué nos señalan estos materiales sobre sus prácticas y orientaciones? Como ya destacamos, el librero francés Claude Joly fue uno de los más constantes emisores de catálogos. Desde fines de la década de 1860 y hasta inicios del siglo XX, cuando los hermanos Prudent le suceden, este librero reporta casi un centenar de catálogos publicados. Le siguen nombres como los de Jacobo Peuser, Alfredo Brédahl, Manuel Reñé y Félix Lajouane, librero este último sobre la que volveremos luego. Por encima de todos aparece, sin
16. La larga cifra colombiana para el decenio de 1910 responde a la actividad de casi un único catálogo, la Revista bibliográfica de la Librería de José Vicente Mogollón de Barranquilla, que reporta 152 números entre 1912 y 1918. 
embargo, otro librero de origen migrante, el danés Louis Jacobsen, con 128 catálogos registrados, la mayor parte correspondientes a su Catalogue mensuel de la Librairie Française distribué par La Librería Europea. Impreso en París por la Typographie de Paul Schmidt, este folleto hacía explícitas sus relaciones con el gremio editorial francés y sería, junto con el Boletín bibliográfico de Joly -impreso también en París por la Typographie de Ch. Lahure-, el órgano de librería más constante entre las décadas de 1870 y 1880.

Tabla 1. Mayores productores de catálogos, 1860-1910

\begin{tabular}{lc}
\hline Librería/Librero & Catálogos \\
\hline Librería Europea de L. Jacobsen y Cía. & 128 \\
Librería de Claude M. Joly (Joly; Prudent Hnos. y Moetzel) & 97 \\
Librería de Jacobo Peuser & 92 \\
Librería Brédhal (Alfredo Brédhal) & 36 \\
Librería y Papelería La Publicidad (Manuel Reñé) & 24 \\
Librairie Française (A. Espiasse y J. Escary; Joseph Escary) & 21 \\
Librería de Ángel Estrada & 20 \\
Librairie Générale de Félix Lajouane (Librería Nacional de J. Lajouane) & 17 \\
Librería Italiana (Alfredo Cantiello) & 16 \\
Librería de Mayo (Carlos Casavalle; Casavalle Hijo) & 13 \\
Librería del Colegio (Igón Hnos; Cabaut \& Cía.) & 11 \\
Librería Rivadavia (Gustavo Mendesky) & 10 \\
\hline
\end{tabular}

Fuente: Elaboración propia a partir de la base de datos CLL

Ahora bien, la producción de catálogos por parte de Félix Lajouane, otro notable librero francés, merece abrir un paréntesis, ya que su posición en la tabla es discutible. Según la numeración que incorporan las portadas de dos de sus catálogos generales, Lajouane habría publicado a septiembre de 1887 su catálogo No. 54, y en abril de 1897 el No. 65. El intervalo entre estas piezas supone una cifra de ocho catálogos publicados en diez años. La falta de datos adicionales impide, no obstante, fijar claramente el comienzo de estas publicaciones y hacer estimaciones confiables sobre su periodicidad, por lo que no se ha ingresado en la base de datos ningún registro especular con relación a estos hallazgos.

A esta dificultad se suma el estudio de Edwin Borchard (1917: 17), quien afirma que el Boletín bibliográfico emitido por Juan Lajouane, hijo y sucesor de Félix, contaba a diciembre de 1916 con 229 números. Este dato llevaría el origen de este folleto mensual hacia 1898, pero por el Boletín de la Biblioteca Pública en La Plata sabemos que el primer número de un Boletín bibliográfico de libros argentinos y extranjeros de Félix Lajouane fue publicado en enero de 1901, boletín que de haberse mantenido hasta 1916 reportaría unas 180 entregas. De todas formas, no hay certeza de que este Boletín se haya prolongado tanto y sea el mismo al que refiere Borchard, lo que implicaría, de hecho, que este tuvo números extraordinarios y cambios en su denominación. De encontrarse piezas que permitan confirmar la constancia de estos boletines y asegurar su conteo fino, los Lajouane no solamente pasarían a encabezar la tabla de productores, sino que elevarían sustancialmente la presencia de estos materiales en dos décadas que muestran, por ahora, tendencias a la baja. 


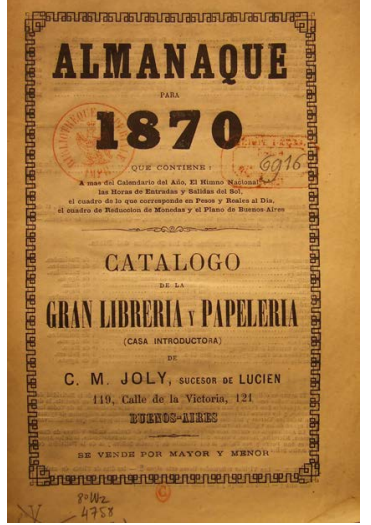

Imagen 4. Portada de Almanaque para 1870 y Catálogo de la gran librería y papelería de C.M. Joly

Fuente: BnF, cota: $8-W Z-4758$
Frente a la dificultad que se patenta con los Lajouane, los casos de Jacobsen y Joly no pueden dejar de resaltarse, pues los datos de sus catálogos permiten complejizar sus figuras. Aunque el estudio pionero de Buonocore (1974) subraya bien algunas de las características de ambos libreros, sobre todo en relación con su papel en la extensión del oficio en Buenos Aires y la construcción de ofertas literarias cosmopolitas y atractivas para intelectuales y académicos, este no avanza propiamente sobre las formas de sus vínculos con el medio editorial europeo ni los porqués de sus capacidades.

En lo fundamental, y una vez cruzada con otras fuentes, la información proporcionada por los catálogos favorece una reconstrucción más detallada de los itinerarios de los libreros, así como de sus estrategias y redes de abastecimiento. El caso de Joly es especialmente dúctil como ejemplo. De acuerdo con Buonocore, su itinerario librero comenzó en 1858 con la compra la Librería Central a su connacional H. Lucien, uno de los viejos favorecidos con el remate de la Librería de Marcos Sastre. Los catálogos demuestran, sin embargo, que la actividad librera de Joly comenzó hacia julio de 1869, momento en que aparece su primer Boletín bibliográfico, un folleto de 4 páginas que logró extenderse, que sepamos, hasta junio de $1876^{17}$. Es también en 1869 que debuta su Almanaque para 1870 y Catálogo de la gran librería y papelería de C.M. Joly, publicación anual que traspasaría el siglo (Imagen 4).

Gracias a estos materiales puede afirmarse que la relación entre Joly y Lucien no finalizó con la compra de la librería. Por un lado, es probable que el apellido Lucien contaba con buena recordación entre los públicos lectores locales y el movimiento comercial bonaerense, pues Joly se presentó siempre como su "sucesor" en boletines y almanaques desde 1869 hasta 1875 . Un periodo de "transición" notablemente largo. Por otro lado, fue posible establecer que Lucien pasó a operar como intermediario de Joly ante los editores parisinos. A diferencia de la mayoría de los libreros de América Latina, Joly era capaz de hacerse propaganda en las revistas que seguían la producción editorial francesa, como el Journal général de limprimerie et de la librairie, medio en el que uno de sus anuncios presentó justamente a H.C. Lucien como "son représentant à Paris (81, boulevard Saint-Germain)"18, y quien "fera parvenir à BuénosAyres les Propesctus, Catalogues, Spécimens de Livres Journaux et Publications de toutes sourtes que Messieurs les Editeurs voudront bien lui envoyer" (1870: 520).

Dada la similitud entre las prácticas de Joly y Jacobsen, especialmente en cuanto al uso del formato boletín-que en cierto modo documenta su conocimiento de las tradiciones propagandísticas del oficio-, es probable que el librero danés contara con conexiones similares en París, esto es, cercanas a las grandes casas editoriales y dueñas de una reputación suficiente para adelantar negocios a distancia. No obstante, sus
17. Estos catálogos se encuentran resguardados en la Bibliothèque nationale de France y pueden identificarse con la cota 8-Q-252.

18. La dirección de Lucien lo ubicaba, de hecho, muy cerca de la sede principal de Hachette, que se situaba en el 79 del boulevard SaintGermain y era ya por entonces la principal casa editora de Francia. 
capacidades de conexión parecen haber sido mucho mayores. Uno de sus primeros catálogos consultado, datado en 1874, cuando todavía ejercía en compañía de Théodor Söderstedt, afirmaba en sus preliminares que la librería contaba con agentes "activos y experimentados" en "Alemania, Francia, Inglaterra, Italia y España".

Los contenidos del catálogo así lo demostraban. Su primera sección se dedicó a listar publicaciones y periódicos franceses y belgas, mientras que su apartado de colecciones presentó a la "Biblioteca de Maravillas" como aquella traducida bajo la dirección de Mariano Urrabieta, o sea, la misma impulsada desde París por Hachette para el mercado hispanoamericano. Este catálogo incluyó además una "Colección de Autores españoles de F.A. Brockhaus", el sello alemán que poco después amplió su proyección en el continente. Pocos años más tarde, y en paralelo a la continua emisión de boletines en lengua francesa, Jacobsen dio cuenta de un Catalogue of new books, received by last mail, being part of the stock of the Libreria Europea (1879), hoy depositado en la biblioteca del Museo Mitre y que presumía de sus conexiones inglesas.

Entrada la década de 1880, los catálogos de Jacobsen continuarán revelando su geografía de abastecimiento. Una extensa pieza de 720 páginas, impresa en Barcelona, en 1885 , fue presentada como solo concerniente a la "Sección de obras en español, modernas, impresas en Europa ó en América", lo que en la práctica daba a entender que la librería estaba dividida en departamentos separados por idiomas. Gracias a la investigación de Ceballos Viro (2007), sabemos que la sección dedicada a las obras en alemán fue regentada por un Carl L. Annecke, lo que permite imaginar que las demás eran también arbitradas por dependientes experimentados. Las páginas finales de este catálogo, insertas probablemente en Buenos Aires, anunciaban además que la Librería tenía "Libros Chilenos - Libros Venezolanos", y que estaba en capacidad de traer publicaciones de "todas las repúblicas Sud-Americanas", incluida Brasil.

Este último aspecto, que dirige la mirada hacia las redes propiamente latinoamericanas de los libreros, permite destacar un último itinerario, el de Carlos Casavalle. A pesar de no haber sido un gran productor de catálogos, su cercanía con la élite letrada porteña, iniciada de la mano de Juan María Gutiérrez a mediados del siglo XIX, le permitió concebir órganos que resaltaban por su rigurosidad bibliográfica y orientación "americanista". Su Boletín bibliográfico sudamericano (1870) fue el ejemplo más acabado, pues junto con incluir las obras de Mitre, Gutiérrez o Echeverría que él mismo editaba, ofreció las de historiadores peruanos, colombianos, chilenos y uruguayos. Dada la especialidad de estos folletos, muchos lograron una circulación continental, como es el caso de un "Estracto del Catálogo Americano" (c. 1878) hallado en el Archivo Nacional de Chile y cuyo claro objetivo era "circular en los pueblos del Pacífico".

Entrado el siglo XX, el establecimiento mantendría su imagen como un negocio distinto a los demás, uno en el que convergían menos las últimas novedades que los autores y títulos representativos de la historia política e intelectual del país y la región. Un catálogo de 1901 incluirá así un "suplemento de las obras americanas de esta antigua casa" con "libros de verdadera importancia histórica para los anales argentinos", mientras que otro publicado en 1903 afirmaba contener un "índice de periódicos y revistas para los coleccionistas". En esta medida, no sobra relevar que los catálogos de Casavalle se dirigían a un mercado particular, delineado por las necesidades de comunicación e intercambios letrados y los deseos de acumulación de bibliotecas nacionales, coleccionistas privados y libreros anticuarios.

En síntesis, los catálogos de venta de Joly, Jacobsen y Casavalle permiten constatar numerosas conexiones transnacionales y, junto con ellas, explorar la plena incorporación de la geografía latinoamericana al mercado internacional del libro. Los 
ejemplos descritos permiten, en efecto, enfatizar que la producción de catálogos guarda relación con el grado de internacionalización. Como lo muestran también los negocios de Estrada, Lajouane, Cantiello, Brédahl, Escary, Bonmatí, o Peuser, a mayor número de vínculos transnacionales mayor número de libros importados y mayor la necesidad de imprimir catálogos que dieran razón de estos. Las librerías con mayor presencia mediática fueron así aquellas mejor engranadas al mercado internacional, un elemento correlacionado además con el origen migrante de libreros y dependientes y, por supuesto, clave en la modernización del comercio de librería en la Argentina.

\section{La presentación del catálogo y su formato}

La presentación de los catálogos es un último asunto por tocar. Como se planteó páginas atrás, la información recogida en la base de datos evita avanzar sobre muchos elementos de interés, por lo que aquí se exploran solo algunos cambios en las lógicas de presentación asumidas por los catálogos, y lo que estas pueden indicarnos sobre el mercado en el que se desenvolvían. En términos generales, hasta la década de 1860 los catálogos pueden ser descritos como simples listas impresas que presentaban un número de obras en venta ordenado alfabéticamente, bien en virtud del título de los libros o del nombre de sus autores.

Cuando se trataba de listados mayores a 20 páginas, sin embargo, el orden podía presentar también una división por lenguas, como en el caso del catálogo de la Librería de Eudaro Carrasco, o incluso ensayar divisiones por géneros. Con todo, estos catálogos no relucían por su sofisticación. Muchos abreviaban los títulos de las obras y no precisaban siempre sus páginas, tomos ni formatos. La situación fue otra a partir de la década de 1870, y en especial con casos como el de Casavalle, quien además de avanzar en precisión bibliográfica incorporó prospectos, juicios y breves noticias bibliográficas de sus principales títulos en venta, características que poco después replicaron libreros-editores como Félix Lajouane.

Que Casavalle y Lajouane aplicaran criterios de catalogación donde el detalle bibliográfico y el comentario crítico y publicitario se imponían responde, por supuesto, a sus exigentes clientelas, compuestas por figuras letradas preocupadas por el origen, valor y relevancia de las obras que adquirían, y que por sus viajes, vínculos e intercambios transnacionales estaban al corriente de los movimientos del mercado del libro internacional y las convenciones bibliográficas utilizadas por los libreros más famosos. En relación con este último aspecto, las prácticas catalogadoras de estos libreros documentan la transferencia de una modalidad de ordenamiento y publicitación propia a libreros anticuarios como Bernard Quaritch o Karl W. Hiersemann, ambos partícipes del tráfico de libros americanos y conectados con el medio letrado de Buenos Aires ${ }^{19}$.

Ahora bien, una mutación adicional en la presentación de los catálogos, y transversal en el medio, se encuentra en la disposición de los precios. Al parecer, esta práctica no fue regular hasta la década 1870 , y en virtud de las exigencias surgidas por el aparataje institucional asociado con la instrucción pública y las bibliotecas populares. Para mediados de 1872, el redactor del Boletin de las Bibliotecas Populares se quejaba, por ejemplo, de que no se hubiese podido conseguir que los libreros de la ciudad publicaran sus catálogos con precios, un hecho que entorpecía los procesos de adquisición libresca ${ }^{20}$.

Ante este tipo de reclamos y, sin duda también en respuesta a la expansión del gremio y el alza de la competencia, la inserción de los precios comenzó a volverse común. Así lo evidencian los folletos de la Librería Europea de Jacobsen, que desde la década
19. Baste remitir al largo listado de catálogos de ambos libreros contenido en la biblioteca del Museo Mitre o en el intercambio entre Quaritch y Casavalle que se verifica en la colección de autógrafos de este último, resguardada en el Archivo General de la Nación.

20. Véase: Boletín de las Bibliotecas Populares. Publicación Periódica, vol. 2. Buenos Aires, 1872, 171. 
Imagen 5. Portada de Catálogo general de la Librería del Colegio. Buenos Aires: Cabaut y Cía. Libreros-Editores, 1907.

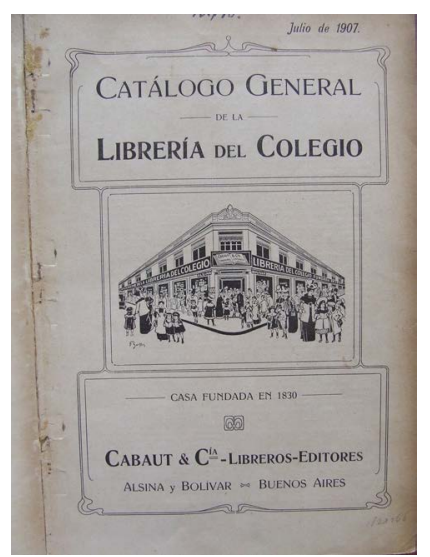

Nota: Ejemplo de las mutaciones de los grandes catálogos, esta pieza de 312 páginas fue impresa en 4 to mayor ( $19 \mathrm{~cm}$.), incorporó índice general y de autores, y fue profusa en el uso de fotografías e ilustraciones, todo al tiempo que expresaba, pese a su calidad, una condición gratuita. Fuente: Biblioteca Nacional de Maestros, colección general. de 1870 empezaron a incluir los precios de los libros en moneda corriente argentina. Casavalle, Lajouane y Reñé hicieron lo propio para esta misma época, e inclusive Joly, quien empezó a exponerlos en sus catálogos generales.

Para finales del siglo XIX es posible observar además un giro en los formatos. Si bien los catálogos generales impresos en $8 \mathrm{vo}$ menor evidencian una larga constancia y comienzan inclusive a insertar ilustraciones, algo patente en los gruesos catálogos de la Librería del Colegio bajo Cabaut y Cía, estos empezaron a compartir el espacio comercial con folletos mucho más modestos, volátiles y despreocupados de las convenciones bibliográficas (Imagen 5). La normalización de este tipo de catálogos periódicos, casi siempre gratuitos y publicados bajo la denominación de boletín señala algunas cuestiones de importancia.

Por un lado, estos ilustran el ímpetu tomado por los libreros en materia publicitaria. A diferencia del catálogo general, que puede adquirir un precio por su volumen o sofisticación, el pequeño boletín circula de forma fácil y constante por calles y correos, garantizando la visibilidad de las librerías y la difusión de su surtido en un amplio y horizontal radio de lectores. Por el otro, la mayor frecuencia de estos boletines abre preguntas sobre la relevancia y utilidad del catálogo general. Aunque no dejan de producirse, es indudable que su vigencia en materia de precios o disponibilidad de títulos era difícil de mantener en un espacio comercial creciente y además sensible a las crisis económicas y la inflación. En otras palabras, el uso del boletín podía dar un margen de maniobra al librero para actualizar rápidamente su oferta.

A pesar de que el uso del boletín despega temprano en la Argentina, gracias a la acción de Joly, Jacobsen y otros, su multiplicación se dispara en el entre siglos, periodo en el que prospera el Boletín bibliográfico argentino. Crónica mensual del movimiento intelectual en la República Argentina y catálogo general de libros americanos y europeos de Jacobo Peuser, con 87 ediciones entre 1895 y 1902; pero donde también surgen el folleto $\mathrm{La}$ Biblioteca. Catálogo de obras nacionales y extranjeras de la Librería Brédahl, con 36 entre 1900 y 1903; la revista mensual Le Livre de la Librería Française de Joseph Escary, que cubrió los años de 1902 y 1903, y los referidos boletines de los Lajouane. Ad portas de la época del Centenario, esta suerte de eclosión del boletín de venta conecta con el momento que Alejandro Parada (2007a: 117-120) describe como de "ávida lectura de los catálogos", asociado a un apogeo en el uso de estos efímeros publicitarios por toda clase de tiendas y negocios.

Más allá de estos elementos, y como lo recuerda Frédéric Barbier (2009), el uso del boletín entre los libreros remite a una tradición de larga data en la historia del 
oficio, asociada con las prácticas de las librerías internacionales y sus especializadas capacidades de intermediación. Que libreros de origen francés, danés y alemán recurran a este tipo de catálogos en Buenos Aires no es, pues, un fenómeno singular, pero sí permite aportar otra característica al consabido cosmopolitismo de la geografía del libro porteño, al tiempo que permite reconocer la evolución de las estrategias publicitarias que envolvían al comercio de librería en la Argentina.

\section{Consideraciones finales}

Más allá de su condición de fuente para la historia del comercio del libro, los catálogos de librería conforman un objeto de investigación valioso en la reconstrucción de la historia de las librerías, sus prácticas y mutaciones como negocios culturales. Como se ha mostrado, su naturaleza bibliográfica, comercial y publicitaria les convierte en producciones idóneas para acercarse a la posición de los libreros en el mercado e identificar sus conexiones transnacionales, geografías de acción y modalidades de venta. Como lo recalca Mollier (1996: 332), los catálogos pueden ayudar incluso a explorar los cambios en las relaciones entre autores y libreros, y entre autores y editores, pues estas nunca son fijas, sino que mutan según los tiempos y las coyunturas.

En lo que concierne a este estudio, se ha podido demostrar, particularmente, que los catálogos suministran rutas para analizar la evolución de ramo librero, a la vez que ayudan a marcar los momentos de expansión del oficio, denotan giros hacia la especialización o hacia el comercio general y mayorista, e incluso señalan mutaciones materiales que revelan cambios en las estrategias comerciales y la agudización de la competencia dentro del ramo. Del mismo modo, se ha mostrado aquí que los catálogos permiten rastrear y reconstruir los vínculos que se establecían entre libreros de distintos países y que posibilitaban, en no pocos casos, la conformación de ofertas relativamente comunes en muy distintas capitales. En esta medida, el catálogo de librería se eleva como una fuente y objeto ineludible para la historia transnacional del libro y la edición.

Este estudio ha permitido advertir, asimismo, que los catálogos de librería tuvieron un lugar en la formación de una cultura de lo impreso en la Argentina, gracias a su extendida, constante y multiforme circulación en el espacio público, pero sobre todo a su muy probable consulta por parte de innumerables lectores que, si bien no siempre podían llegar a ser clientes, podían sí estar al corriente de las novedades del mercado. Dicho de otro modo, el catálogo, como producto impreso, integró el aluvión de diarios, revistas, colecciones, cartillas, almanaques y sueltos de todo tipo que atravesó el momento de entre siglos y delineó el paisaje librero-editorial de la época del Centenario.

Los catálogos siguen ocupando, de todos modos, un lugar marginal en los estudios sobre el libro y la edición en América Latina. Su examen contrae, ciertamente, dificultades, pues el investigador debe enfrentarse tanto a su dispersión en archivos y bibliotecas, como a clasificaciones que dificultan su identificación. Pero antes que desanimar el interés por estos materiales, sus contenidos, productores y espacios de circulación, este estudio espera haber alertado sobre la necesidad de enriquecer y visibilizar las colecciones hoy existentes, e imponer el desafío de escudriñarlas. 


\section{Q Referencias bibliográficas}

Agustí, Lluís; Mònica Baró y Pedro Rueda Ramírez, eds. 2018. Edición y propaganda del libro. Las estrategias publicitarias en España e Hispanoamérica (siglos $X V I I-X X)$. Valencia: Calambur.

»Almanaque comercial y guía de forasteros para el Estado de Buenos Aires. 1855. Buenos Aires: Imprenta de "La Tribuna”.

» Barbier, Frédéric. 2009. Pour une anthropologie culturelle des libraires: note sur la librairie savante à Paris au XIXe siècle. En Histoire et civilisation du libre. Vol. 5, 101-120.

»Boletín de la Biblioteca Pública de la Provincia de Buenos Aires en la ciudad de La Plata. 1899-1905. La Plata: Talleres de Publicaciones del Museo.

»Borchard, Edwin M. 1917. Guide to the Law and Legal Literature of Argentina, Brazil, and Chile. Washington: Government Printing Office.

"Buonocore, Domingo. 1974. Libreros, editores e impresores de Buenos Aires. Esbozo para una historia del libro argentino. Buenos Aires: Bowker Editores Argentina.

»Ceballos Viro, Álvaro. 2007. La implantación de la librería alemana en España e Hispanoamérica de 1850 a 1900. En Schmelzer D. et al., eds. Handeln und Verhandeln. Beiträge zum 22. Forum Junge Romanistik (Regensburg, 07.-10.6.2006). Bonn: Romanistischer Verlag, 453-472.

»Charon, Annie y Elisabeth Parinet, eds. 200o. Les ventes de livres et leurs catalogues XVIle-XXe siècle. Paris: École des chartes.

»Chartier, Roger. 2012. Cardenio entre Cervantes y Shakespeare. Historia de una obra perdida. Buenos Aires: Gedisa.

»Coppens, Christian y Angela Nuovo. 2018. Printed catalogues of booksellers as a source for the history of the book trade. En JLIS.it, Vol. 9, no. 2. 166-178. < http://dx.doi.org/10.4403/jlis.it-12465>

»Criscuolo, Eduardo Luis. 200o. Bibliografía de la ciudad de Buenos Aires. Buenos Aires: Instituto Histórico.

»De la Fuente, Diego. 1872. Primer censo de la República Argentina verificado en los días 15, 16 y 17 de septiembre de 1869. Buenos Aires: Imprenta del Porvenir.

»Granata, Giovanna y Angela Nuovo. 2018. Selling \& Collecting: Printed Book Sale Catalogues and Private Libraries in Early Modern Europe. Macerata: Eum Edizioni Università di Macerata.

" Journal général de l'imprimerie et de la librairie. Deuxième serie. Tome XIV. Deuxième partie: Chronique. 1870. París: Au Cercle d l'imprimerie, de la librairie et de la papeterie.

»Kunz, Hugo. 1886. Gran guía de la ciudad de Buenos Aires. Buenos Aires: Hugo Kunz y Cía.

"Lima González Bonorino, Jorge F. 2005. La ciudad de Buenos Aires y sus habitantes 1860-1870 a través del Catastro de Beare y el Censo Poblacional. Buenos Aires: Instituto Histórico de la Ciudad de Buenos Aires. 
» La Literatura Argentina. Revista bibliográfica. 1932. Buenos Aires: Talleres Gráficos Argentinos L. J. Rosso.

"Mollier, Jean-Yves. 1996. L'histoire de l'édition, une histoireà vocation globalisante. En Revue d'histoire Moderne et Contemporaine. Vol. 43, no. 2, 329-348.<https:// www.persee.fr/doc/rhmc_0048-8003_1996_num_43_2_1820> [Consulta: 10 mayo 2020].

"Navarro Viola, Alberto. 1882. Anuario Bibliográfico de la República Argentina. Año III-1881. Buenos Aires: Imp. Biedma.

» Nuovo, Angela. 2013. The book trade in the Italian Renaissance. Leiden, Boston: Brill.

"Parada, Alejandro E. 2002. El orden y la memoria en una librería porteña de 1829: el Catálogo de la Librería Duportail Hermanos. En Información, Cultura y Sociedad. No. 7, 9-8o. <http://revistascientificas.filo.uba.ar/index.php/ICS/ article/view/967> [Consulta: 10 mayo 2020].

"Parada, Alejandro E. 2007a. Cuando los lectores nos susurran: libros, lecturas, bibliotecas, sociedad y prácticas editoriales en la Argentina. Buenos Aires: Universidad de Buenos Aires. Facultad de Filosofía y Letras. Instituto de Investigaciones Bibliotecológicas INIBI. <http://publicaciones.filo.uba.ar/cuando-loslectores-nos-susurran $>$ [Consulta: 10 mayo 2020].

"Parada, Alejandro E. 2007b. Los itinerarios lectores de un librero cultural. El "Catálogo" de la Librería Argentina de Marcos Sastre (1835). En Boletín de La Academia Argentina de Letras. Vol. 72, no. 289-290, 215-272. <http://www.catalogoweb.com.ar/biblioteca-digital/b2007.html> [Consulta: 10 mayo 2020].

» Pastormelo, Sergio Carlos. 2005. El nacimiento de un mercado editorial en Buenos Aires, 1880-1890. En Orbis Tertius. Vol. 10, no. 11, 143-158. <https://www. orbistertius.unlp.edu.ar/article/view/OTv1on11do2> [Consulta: 10 mayo 2020].

»Planas, Javier. 2017. Libros, lectores y sociabilidades de lectura. Una historia de los orígenes de las bibliotecas populares en la Argentina. Buenos Aires: Ampersand.

» Rueda Ramírez, Pedro y Lluís Agustí. 2016. La publicidad del libro en el mundo hispánico (siglos XVII-XX): los catálogos de venta de libreros y editores. Madrid: Calambur.

"Sabato, Hilda. 2012. Historia de la Argentina, 1852-1890. Buenos Aires: Siglo Veintiuno Editores.

»Sorá, Gustavo. 2011. El libro y la edición en la Argentina: libros para todos y modelo hispanoamericano. En Políticas de la Memoria. No. 10-11-12, 125142. <https://ojs.politicasdelamemoria.cedinci.org/index.php/PM/article/ view/527> [Consulta: 10 mayo 2020].

" Torrico, Milenka. 2020. Librería “La Universitaria" de los hermanos Arnó: Un estudio sobre el comercio del libro en La Paz a principios del siglo XX. Bogotá: Instituto Caro y Cuervo, Facultad Seminario Andrés Bello, 2020. Tesis de Maestría en Estudios Editoriales. 
